\title{
Erratum
}

\section{Erratum to: Src Is Dephosphorylated at Tyrosine 530 in Human Colon}

Carcinomas

Shudong Zhu* , Jeffrey D Bjorge, Donald J Fujita

Department of Biochemistry and Molecular Biology, and Southern Alberta Cancer Research Institute,

University of Calgary, Calgary, Alberta, Canada

DOI: 10.1007/s11670-011-0324-8

(c) Chinese Anti-Cancer Association and Springer-Verlag Berlin Heidelberg 2011

Erratum to: Chin J Cancer Res 23(3):229-231, 2011

DOI: $10.1007 /$ s11670-011-0229-6

The first author name was misspelled in the original version of this article. The name "Shu-dong Zhu" should be corrected to "Shudong Zhu".

The online version of the original article can be found at http://dx.doi.org/10.1007/s11670-011-0229-6 